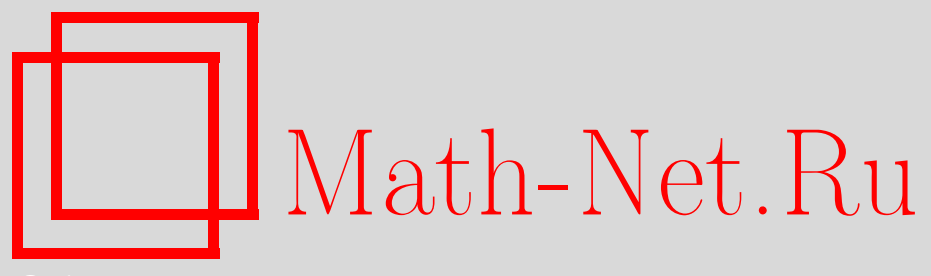

А. М. Райгородский, О структуре графов расстояний, имеющих большое хроматическое число, Матем. заметки, 2006, том 80, выпуск 3, 473-475

DOI: https://doi.org/10.4213/mzm2836

Использование Общероссийского математического портала Math-Net.Ru подразумевает, что вы прочитали и согласны с пользовательским соглашением http://www . mathnet.ru/rus/agreement

Параметры загрузки:

IP: 3.80 .181 .102

26 апреля 2023 г., 13:34:42

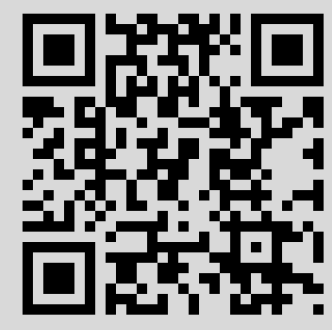




\section{О СТРУКТУРЕ ГРАФОВ РАССТОЯНИЙ, ИМЕЮЩИХ БОЛЬШОЕ ХРОМАТИЧЕСКОЕ ЧИСЛО}

\section{А. М. Райгородский}

1. Постановка задачи и формулировки результатов. В 50-е годы XX века Е. Нелсон предложил исследовать минимальное число $\chi\left(\mathbb{R}^{n}\right)$ цветов, в которые можно так раскрасить все точки вещественного евклидова пространства, чтобы между точками одного цвета не было расстояния 1 (см. [1]). Это число, называемое хроматическим числом пространства, стало знаменитым благодаря Г. Хадвигеру (см. [2]), который сам занимался смежными вопросами еще в 1944 г. (см. [3]), и П. Эрдёшу, для которого постановка аналогичных задач была весьма характерна. Термин "хроматическое число" в данном контексте вовсе не случаен. Дело в том, что задачу Нелсона-Эрдёша-Хадвигера ничего не стоит переформулировать на языке теории графов, полагая $\chi\left(\mathbb{R}^{n}\right)=\chi\left(\mathscr{G}_{n}\right)$, где $\mathscr{G}_{n}=\left(\mathscr{V}_{n}, \mathscr{E}_{n}\right)$ - граф с множеством вершин $\mathscr{V}_{n}=\mathbb{R}^{n}$, множеством ребер

$$
\mathscr{E}_{n}=\{(\boldsymbol{x}, \boldsymbol{y}) \in \mathscr{V} \times \mathscr{V}:|\boldsymbol{x}-\boldsymbol{y}|=1\}
$$

$(|\boldsymbol{x}-\boldsymbol{y}|$ - обычное евклидово расстояние между векторами) и хроматическим числом $\chi\left(\mathscr{G}_{n}\right)$. Конечно, граф $\mathscr{G}_{n}$ "ужасен", ведь множество его вершин континуально, и априори не вполне ясно, как можно работать с его хроматическим числом. Тут, однако, на помощь приходит замечательная теорема П. Эрдёша и Н. де Брёйна: если хроматическое число какого-либо графа конечно, то оно достигается на некотором конечном подграфе исходного графа (см. [4]). Таким образом, если показать, что $\chi\left(\mathbb{R}^{n}\right)<\infty$, то дальше про континуум вершин можно забыть и манипулировать исключительно так называемыми конечными графами расстояний, т.е. подграфами графа $\mathscr{G}_{n}$. Практически очевиден тот факт, например, что $\chi\left(\mathbb{R}^{n}\right) \leqslant(c n)^{n}, c>0$, так что заведомо все в порядке. Более того, известно, что $\chi\left(\mathbb{R}^{n}\right) \leqslant(3+o(1))^{n}$ (см. [5]), и этот результат никому не удается улучшить с 1972 г. Интересная проблема состоит, стало быть, в отыскании нижних оценок. Впервые их экспоненциальность была установлена П. Франклом и Р. М. Уилсоном, которые в 1981 г. обосновали неравенство $\chi\left(\mathbb{R}^{n}\right) \geqslant(1.207 \ldots+o(1))^{n}$ (см. [6]). Единственное улучшение оценки Франкла-Уилсона было найдено автором в работе [7]: $\chi\left(\mathbb{R}^{n}\right) \geqslant(1.239 \ldots+o(1))^{n}$.

В дальнейшем нас будет в первую очередь волновать структура тех графов расстояний, на которых достигаются наилучшие экспоненциальные оценки хроматического числа пространства. K ее обсуждению мы сейчас и перейдем. Заметим лишь, что в связи с проблемой Нелсона-Эрдёша-Хадвигера получено огромное количество результатов, и многие из них можно найти в обзорах [8] и [9].

Наиболее просто устроены графы расстояний, за счет которых получается неравенство $\chi\left(\mathbb{R}^{n}\right) \geqslant(1.207 \ldots+o(1))^{n}$, принадлежащее Франклу и Уилсону. Однако уже с ними ясно не все. Посуществу они имеют вид $G=(V, E)$, где

$$
V \subseteq \Sigma=\left\{\boldsymbol{x}=\left(x_{1}, \ldots, x_{n}\right): x_{i} \in\{0,1\},\left|\left\{i: x_{i}=1\right\}\right|=l\right\}
$$

с некоторым $l \in[0, n]$, а

$$
E=\{(\boldsymbol{x}, \boldsymbol{y}) \in V \times V:|\boldsymbol{x}-\boldsymbol{y}|=a\} .
$$

Работа выполнена при финансовой поддержке гранта Президента РФ № МК-3130.2004.1, программы поддержки Ведущих научных школ, грант № НШ-136.2003.1, и фонда ИНТАС, грант № 03-51-5070.

(C) А. М. РАйгородский, 2006 
Здесь мы вольны варьировать параметры $l$ и $a$. Заметим, кстати, что формально граф $G$ не обязан являться подграфом в $\mathscr{G}_{n}$, ведь у $\mathscr{G}_{n}$ ребра задаются парами точек на расстоянии 1 , а не $a$. Однако хроматическое число пространства инвариантно относительно величины "запрещенного" расстояния (запрещенного между точками одного цвета), и потому единица была взята нами исключительно для удобства первоначального определения.

У Франкла и Уилсона оптимальной оказывалась ситуация, когда $l \sim(1-\sqrt{2} / 2) n$, $a \sim \sqrt{l}$. При таких значениях параметров оценка как раз и имела вид

$$
\chi\left(\mathbb{R}^{n}\right) \geqslant \chi(G) \geqslant(1.207 \ldots+o(1))^{n}
$$

с другими же параметрами оценка была гораздо слабее. Возникает естественный вопрос: так ли уж необходимо, чтобы граф расстояний имел именно упомянутую структуру $(l \sim$ $(1-\sqrt{2} / 2) n, a \sim \sqrt{l})$, дабы его хроматическое число было ограничено снизу величиной $(1.207 \ldots+o(1))^{n} ?$

Формализовать задачу можно так. Пусть $\mathfrak{G}_{1}=\mathfrak{G}_{1}(n ; 0,1 ; n-l, l)$ - класс всех графов расстояний $G=(V, E)$, у которых $V \subseteq \Sigma$ (в каждой вершине - векторе из $\mathbb{R}^{n}$ - ровно $n-l$ нулей и $l$ единиц), а $E$ имеет вид (1) с некоторым (произвольным) $a$. Тем самым, мы получаем как бы две "степени свободы" внутри класса: $V$ любое, хотя структура его элементов задана наперед, и $а$ любое в разумных пределах (бессмысленно брать $a$, большее величины максимального расстояния в $\Sigma$, и пр.). Положим теперь

$$
\chi_{1}=\chi_{1}(n ; 0,1 ; n-l, l)=\max _{G \in \mathfrak{G}_{1}} \chi(G)
$$

Понятно, что в новых терминах $\chi_{1}(n ; 0,1 ; n-l, l) \geqslant(1.207 \ldots+o(1))^{n}$, коль скоро $l \sim$ $(1-\sqrt{2} / 2) n($ Франкл-Уилсон), а при других $l$ такого неравенства пока нет. Стало быть, максимально точные нижние оценки величины $\chi_{1}$ при разных $l$ и будут свидетельствовать о структуре графа расстояний с большим хроматическим числом.

Мы будем считать, что $l \asymp n$. На первый взгляд, такое ограничение кажется существенным, но, на самом деле, если $l=o(n)$, то верхние оценки $\chi_{1}$, принадлежащие автору (см. [10], [11]), субэкспоненциальны, и говорить о величинах типа $(1.207 \ldots+o(1))^{n}$ заведомо не приходится. Итак, пусть $l \sim l_{0} n, l_{0} \in(0,1 / 2)$, a $t=\left[t_{0} n\right]<l$, где $t_{0} \in\left(0, l_{0}\right)$. Определим $p$ как минимальное простое число, такое, что $l-2 p<t$. Поскольку $l-t \asymp n$, величина $p=p(l, t)$ асимптотически равна $(l-t) / 2$ (см. [12]). Известно, что $C_{n}^{l-t}=\left(v_{0}+o(1)\right)^{n}$, где $v_{0}=v_{0}(l, t)$ - константа, большая единицы. Имеет место новая

Теорема. Выполнена оценка

$$
\chi_{1} \geqslant \max _{t} \frac{\left(v_{0}(l, t)+o(1)\right)^{n}}{C_{n}^{p(l, t)-1}} .
$$

По большому счету, в теореме речь идет об отношении $C_{n}^{l-t} / C_{n}^{[(l-t) / 2]}$, которое, как нетрудно проверить, имеет вид $\left(c_{0}+o(1)\right)^{n}, c_{0}=c_{0}(l, t)>1-$ константа: все различия между упомянутой дробью и дробью из формулировки попадают в $o(1)$.

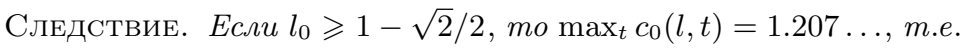

$$
\chi_{1} \geqslant(1.207 \ldots+o(1))^{n} \text {. }
$$

Следствие дает ответ на самый первый наш вопрос: граф расстояний вовсе не обязан иметь структуру, рассматривавшуюся Франклом и Уилсоном, дабы его хроматическое число было надлежащим образом велико. Если раньше при $l=0.5$ мы, скажем, имели $\chi_{1} \geqslant(1.139 \ldots+o(1))^{n}$, то теперь мы имеем $\chi_{1} \geqslant(1.207 \ldots+o(1))^{n}$, и т.д. 
2. Набросок доказательства теоремы и ее следствия. Пусть фиксированы все параметры из формулировки теоремы. Определим натуральное число $r$ из условия

$$
(l-t+1)\left(2+\frac{t-1}{r+1}\right) \leqslant n \leqslant(l-t+1)\left(2+\frac{t-1}{r}\right)
$$

и выберем граф $G_{t}=\left(V_{t}, E_{t}\right) \in \mathfrak{G}_{1}$ следующим образом. Прежде всего возьмем $V_{t} \subset \Sigma$, полагая $V_{t}=\bigcup_{u=t+r}^{t+2 r} V_{t}^{u}$, где

$$
V_{t}^{u}=\left\{\boldsymbol{x}=\left(x_{1}, \ldots, x_{n}\right) \in \Sigma:\left|\left\{i \leqslant t+2 r: x_{i}=1\right\}\right|=u\right\} .
$$

Нетрудно проверить, что скалярное произведение любых двух векторов из $V_{t}$ не может быть меньше $t$. Более того, стандартные комбинаторные выкладки в сочетании с формулой Стирлинга показывают, что $\left|V_{t}\right|=\left(v_{0}(l, t)+o(1)\right)^{n}$. Наконец, результаты работы [13] свидетельствуют о том, что если попарные скалярные произведения векторов в какомлибо $V \subset \Sigma$ ведут себя так же, как и в $V_{t}$ (оцениваются снизу величиной $t$ ), то $|V| \leqslant\left|V_{t}\right|$, а это в конечном итоге означает, что $V_{t}$ построено оптимально. Рассмотрим далее

$$
E_{t}=\left\{(\boldsymbol{x}, \boldsymbol{y}) \in V_{t} \times V_{t}:|\boldsymbol{x}-\boldsymbol{y}|=\sqrt{2 p}\right\} .
$$

Несложная модификация линейно-алгебраического метода из [6] влечет неравенство $\alpha\left(G_{t}\right) \leqslant C_{n}^{p-1}$, где $\alpha\left(G_{t}\right)$ - число независимости нашего графа. Стало быть,

$$
\chi_{1} \geqslant \chi\left(G_{t}\right) \geqslant \frac{\left|V_{t}\right|}{\alpha\left(G_{t}\right)} \geqslant \frac{\left(v_{0}(l, t)+o(1)\right)^{n}}{C_{n}^{p(l, t)-1}},
$$

и набросок доказательства теоремы завершен. Следствие же получается за счет оптимизации установленного неравенства.

\section{СПИСОК ЦИТИРОВАННОЙ ЛИТЕРАТУРЫ}

[1] A. Soifer, Mathematical Coloring Book, Center for Excellence in Mathematical Education, Colorado Springs, CO, 1997. [2] H. Hadwiger, Elemente der Math., 16 (1961), 103-104. [3] H. Hadwiger, Portugaliae Math., 4 (1944), 140-144. [4] N. G. de Bruijn, P. Erdős, Nederl. Akad. Wetensch. Proc. Ser. A, 54:5 (1951), 371-373. [5] D. G. Larman, C. A. Rogers, Mathematika, 19 (1972), 1-24. [6] P. Frankl, R. M. Wilson, Combinatorica, 1:4 (1981), 357-368. [7] А. М. Райгородский, УМH, 55:2 (2000), 147-148. [8] А. М. Райгородский, УМH, 56:1 (2001), 107-146. [9] L. A. Székely, Paul Erdős and his Mathematics, II (Budapest, 1999), Bolyai Soc. Math. Stud., 11, János Bolyai Math. Soc., Budapest, 2002, 649-666. [10] А. М. Райгородский, Матем. сб., 193:10 (2002), 139-160. [11] А. М. Райгородский, Изв. РАН. Сер. матем., 69:3 (2005), 81-108. [12] К. Прахар, Распределение простых чисел, Мир, М., 1967. [13] R. Ahlswede, L. H. Khachatrian, European J. Combin., 18:2 (1997), 125-136.

\section{А. М. Райгородский}

Поступило

Московский государственный университет

20.04.2006

им. М. В. Ломоносова

E-mail: araigor@avangard.ru 